\title{
Subject Index Vol. 70, 1996
}

\section{ABO incompatibility 69}

Adenosine 199

ADP 322

Adult respiratory distress syndrome 271 Anemia 218 Anesthesia 311 Anoxia 280

Antioxidant enzymes 116 Apnea 91,359 Appropriate for gestational age 328 Arachidonic acid 235 Asphyxiated piglets 296

Behavioral state 155 Bilirubin encephalopathy 289

$$
\text { - } \quad \text { oxidation } 289
$$

Brain 289

Breathing, regulation 359 Burst-forming unit, erythrocyte 304

C3a 206

Capillary stress failure 349 Carboxyhemoglobin 69 Cardiopulmonary bypass 141 CD4 T cell subset 108 CD8 T cell subset 108 Cerebral blood volume 91

perfusion and metabolism 141

total hemoglobin concentration 91

Cerebrospinal fluid 296 Cholecystokinin 311 Circulating intercellular adhesion molecule-1 206

108 leucocytes, fetal guinea pig

Circulatory adaptation 15 Circumcision, newborn 213 Cocaine 52 Colony-forming unit, erythrocyte

304 Colostrum 339

Competitive quantitative PCR

199 Coombs-positive hemolytic anemia 69 Cord blood 206 Cordocentesis 84 Cortical development 165 Corticosteroids 101 Cranial ultrasound 29 Cytopenia 304

Delivery 311 Development 199,235 DNA amplification 246 Dopamine antagonism, cocaine 52 Doppler ultrasonography 84

Elastase- $\alpha$ i-proteinase inhibitor complex 206 EMLA ${ }^{\circledR} 213$ Endothelin 115 Enteral feeding 265 Environmental stimulation 165 Enzyme 339 Erythropoiesis 218,304 Erythropoietin 218 -, pharmacokinetics 135

Fetal adipose tissue metabolism, pig 41

blood 84

- sampling 84, 193

rat lung 116 Fetus 101

Flow cytometry 322

Gasping 280

Gastrin 60

Gestational age 29 
Glomerular filtration 229

Glucose 296

Growth factor 339

Gut development, pig 60

Haemorrhage 21

Hemolysis 69

Human fetal gammaglutamyl

transferase 193 Hyperinsulinism 296

Immature newborn 349

Incubator 249

Infants 35, 141

Insulin 296

Interleukins 206

Intestinal motility 265

Intestine 339

Intrauterine growth retardation

84 Intravenous immune globulin 69 Intraventricular hemorrhage 29 Iron 218 Isoimmunization, neonatal 69

Jaundice, neonatal 69

Kernicterus 289 Kidney 235

L-NAME 1 Laryngeal reflex 359 Late gestational period 116 Leukomalacia 29 Lipid peroxidation 116 Lipogenesis 41 Lipolysis 41 Lipoprotein lipase 41 Low frequency 249 Luminal digestion 60 Lung development/maturation 116

- ultrastructure 101

Magnetic fields 249

- $\quad$ flux density 249

Maturation 280

Methemoglobin 213

Methemoglobinemia 213

Methylmalonic acid 304

ß2-Microglobulin 35

367

Mitochondria 289

Mitogens 108

Mycobacterium tuberculosis 246

Near infrared spectrophotometry

91 Neonatal asphyxia 296

intensive care unit 249 Neonate(s) 15,322

piglets, cocaine 52 Neurodevelopmental prognosis

328 Neuronal plasticity 165 Newborns 75 Nitric oxide 1,15 Nosocomial infection 271

$(3 / 4$ free radicals/reactive $(3 / 4$ species 116

Ontogeny, adipose tissue metabolism 41

-, circulating leucocytes 108

Osmolal regulation 229

Peripheral circulation, regulation 359

Pharmacodynamics 218 
Phototherapy 249

Physiological pulmonary hypertension 15

Platelet activation 322

Polymerase chain reaction 246

Posterior fossa cerebellum 29

Potassium excretion 229 Premature infant(s) 29, 249 Prematurity 21 Prenatal diagnosis 84,193

Preterm infants 91,265,311

-, erythropoietin 135 Prilocaine 213 Propionic acid 304 Prostaglandins 235 Pulmonary

hemorrhage 349

infections 128

- $\quad$ pathology, respiratory distress

syndrome 21

- $\quad$ surfactant 128

Pulsating bubble surfactometer

271 Pure red cell aplasia 304

Receptors 199 Renal function 35

- $\quad$ hemodynamics 1

SCH23390 52

Selenium 35

Sepsis 206

Severe sequelae 328

Sheep, fetal 155

Single-dose subcutaneous erythropoietin administration 135

Small preterm neonates 271

Socioeconomical cultural factors 328

Sodium excretion 229 Somatostatin 311 Staphylococcus epidermidis 271 Subfractions 271

Surface activity 271

tension measurement 75 Surfactant function 271

replacement therapy 128

- $\quad$ treatment, pulmonary

pathology 21

Surfactant-bacteria interactions

128 Survival 280

Thermoreceptors 265 Thromboxane A2 322 Thyrotropin-releasing hormone

101 Thyroxine 41 Tissue blood flow 52 Toxicity, prilocaine 213 Tracheal aspirate samples 75

Undernutrition 165

Urinary selenium excretion 35

Vascular occlusion pressures 349 Vasoactive agents 1 Venous circulation 84 Very-low-

birthweight infants 265 Vibroacoustic stimulation 155

Water balance 229

368

Subject Index Vol. 70, 1996 